Fabrice Marchal, Kai Nagel

\title{
Computation of Location Choice of Secondary Activities in Transportation Todels with Cooperative Agents
}

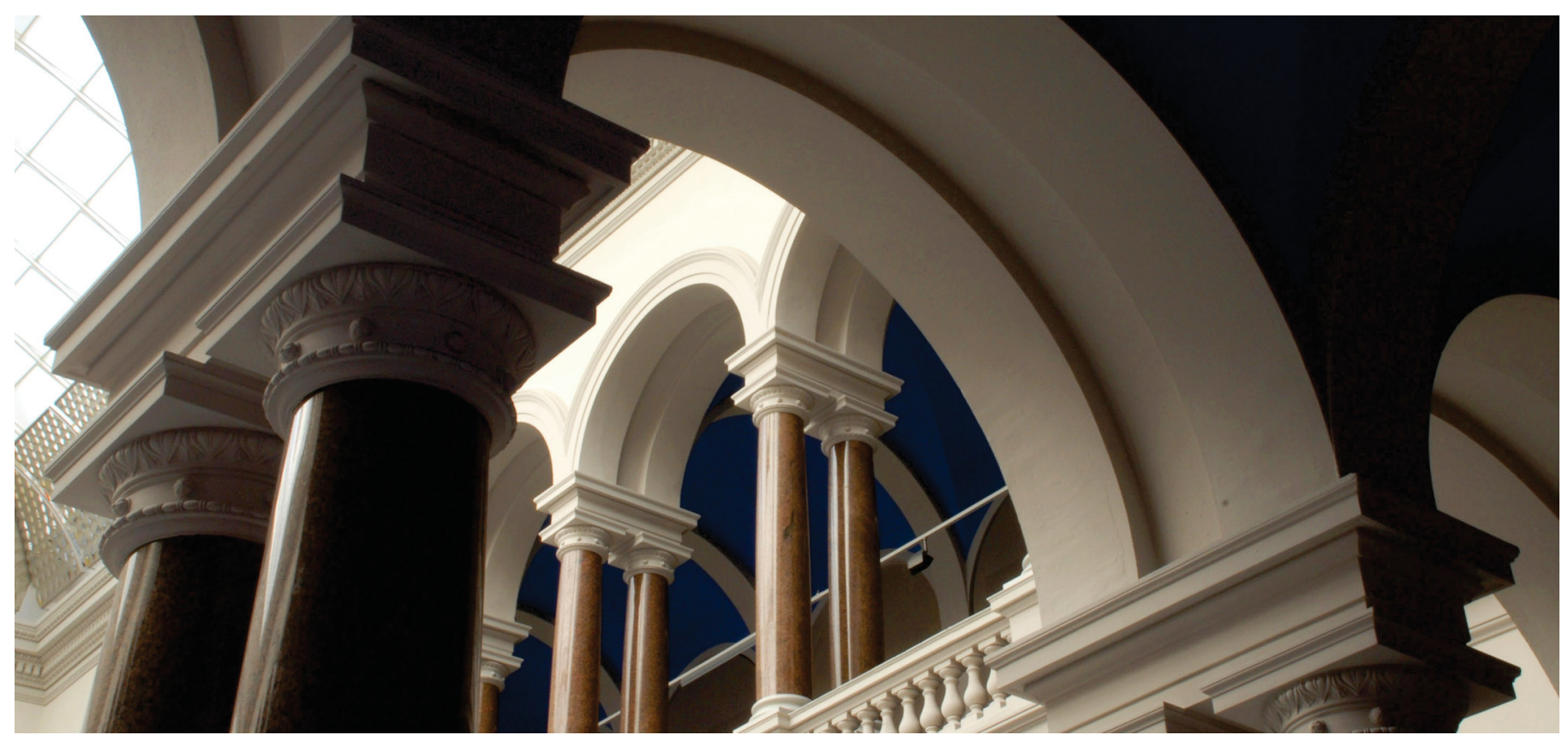

Marchal, F.; Nagel, K. (2005). Computation of Location Choice of Secondary Activities in Transportation Todels with Cooperative Agents. Applications of Agent Technology in Traffic and Transportation, 153-164. https://doi.org/10.1007/3-7643-7363-6_10 


\title{
Computation of location choice of secondary ac- tivities in transportation models with cooperative agents
}

\author{
Fabrice Marchal and Kai Nagel
}

\begin{abstract}
Activity-based models in Transportation Science focus on the description of human trips and activities. We address the modeling of activity location decision for large data sets: given both home and work locations, where do individuals perform so-called secondary activities (e.g shopping)? We propose a model where agents have limited, accurate information about a small subset of the overall spatial environment. Agents are inter-connected by a social network through which they can exchange information. This approach has several advantages: a) it can be faster to find suboptimal solutions to build plausible choice sets b) the learning speed of the overall process is governed by the greediness of the exchange and c) it can provide a useful framework to study the propagation of any newly available information.
\end{abstract}

\section{General context}

Activity-based models in Transportation Science focus on the description of the organization of human activities in time and space. This organization determines the demand for travel, that is the amount of users that the various transportation systems need to accommodate. It is assumed that the demand for travel is derived from the demand for performing activities at specific locations. Obviously, individuals constantly perform some trade-off between enjoying activities that have a high reward value (for instance working at a company in the downtown area) and the time and budget it takes to get to the specific location of these activities. Various operational models such as URBANSIM [10] are available to describe this trade-off for the choice of the home and work locations. It is essentially assumed that users perform a trade-off between rents, travel costs and wages. However, empirical evidence [2] have shown that a significant amount of traffic is generated for other purposes than commuting, often referred to as secondary activities: shopping, leisure, going to social events, etc. 


\section{Problem statement}

Our work intends to model the specific process of the location choice of secondary activities in the case of high resolution data sets. The methodological constraints are that the modeling should be behaviorally sound, compatible with micro-economics foundations and computationally feasible. The temporal dimension (i.e. the scheduling of the activities) is ignored for the time being. We assume that the order of the activities called a plan is given (i.e. getting out from home, going to work, working for eight hours, going for shopping at lunch time, etc.). The physical environment is described by two large data sets that typically originate from Geographical Information Systems (GIS): a) the land-use data and b) the transportation system data. The land usage is a raster-type description that includes the information about the nature of each parcel of the studied area (e.g. housing density, number of shops, type of area: rural, commercial, industrial). The transportation system is a vector-type description of the various transportation modes available (e.g. car, rail, bus) as a network with nodes and links. Nowadays, these data are available at a very high resolution: typical land-use cells are 100 meter square and road networks are described down to 10 meter road sections. The long-term goal of our research is to model entire metropolitan areas microscopically by simulating the individual decision of millions of citizens. Therefore, the problem at hand can be stated as follows: how to simulate the selection of the activity locations of $A=10^{6}$ citizens in a grid that has $C=10^{5}$ cells. Note that the travel times from cell to cell have to be given by external traffic models. Multiagents traffic assignment models such as those developed by the authors $[8,5]$ are now able to predict travel time patterns for large-scale data sets. Therefore, this issue will not be considered here. The generation of travel demand for these models is addressed here.

\section{Micro-Economics foundation}

The standard practice in Transportation Science to approach such problems is to use random utility models (RUM) borrowed from the discrete choice theory of Micro-Economics [3, 6]. These models assume that individual are maximizing their own utility. For instance, the utility to go shopping at a mall located in cell $i$ for a simple plan (i.e. home - shopping - work) is given by

$$
U_{i}=R_{i}-C_{h i}-C_{i w}+\mu \epsilon_{i}=V_{i}+\mu \epsilon_{i},
$$

where $R_{i}$ is the reward associated to shopping at that particular facility that depends on the availability of goods, their prices, etc.; $C_{i h}$ is the travel cost to travel from home to cell $i$; $C_{i w}$ is the travel cost to travel from cell $i$ to work; $\mu$ is a scale factor and $\epsilon_{i}$ is a random variable that is specific to the individual. The latter random utility part captures all the hidden preferences of a specific user for location $i$ that are not accessible to the modeler. By contrast, $V_{i}$ is called the deterministic part of the utility. Under the assumption that $\epsilon_{i}$ are i.i.d. extreme 
value distribution of type I, it can be shown that the probability to choose to go shopping at cell $k$ is given by:

$$
P(k)=P\left(U_{i}<U_{k} \forall i \neq k\right)=\frac{\exp \left(-V_{k} / \mu\right)}{\sum_{i} \exp \left(-V_{i} / \mu\right)}
$$

Since a probability greater than zero is assigned to each potential intermediary stop on a cell, this formulation requires a full enumeration of the possibilities on the spatial grid. For trips that count $S$ intermediary stops, the complexity is $O\left(A C^{S}\right)$ which is not feasible in realistic cases. Initially, RUMs are intended for the description of choices between a finite set of alternatives distinguishable by humans (e.g. car brands). Their application to a discretized continuum (i.e. urban space) remains behaviorally questionable. Still, we believe it is fundamental to keep some compatibility with RUMs because of the huge amount of the literature that has been devoted to developing empirical techniques (e.g. surveys) to calibrate the parameters of those models. Another drawback of RUMs is that they provide only a static representation that does not take into account the temporal dimension of the decision process. RUMs do not model explicitly the learning process performed by human beings and the dynamics due, for instance, to some modification in the land-use patterns.

\section{Multi-agent based approach}

To overcome these limitations, we propose a multi-agent based simulation where each agent (i.e. each simulated citizen) has only limited, accurate information about $N$ cells $(N \ll C)$ called the "memory" of the agent. The intuition is that real humans have limited cognitive abilities and can only consider a small amount of options at the same time. The organization of these options in the mind and the human representation of space is probably far different from "pixels". Nevertheless, we keep that trivial representation for now, as it can be replaced later by a more sophisticate one, for instance that of [1]. Furthermore, we assume that agents are inter-connected by a social network through which they can exchange information about their respective subsets. Each agent is socially connected to $K$ acquaintances or "kins". The simulation is iterative and each round has four stages: evaluation, socialization, exchange and exploration.

Evaluation: each agent performs the location choice of the intermediary stops based on his own private information. The choice can be deterministic (the best cells are selected from the memory of the agent) or probabilistic (a RUM is applied to the finite set limited to the memory of the agent). The computing load for building and storing the travel plans is $O\left(A N^{S}\right)$. At this point, the plans are fed in some traffic model which is run to compute the delays incurred due to traffic congestion. These penalties are to be used in the next round of evaluation. 
Socialization: social connections are created and deleted dynamically. The deletion mechanism is a simple exponential decay. The creation mechanism is a spatial reinforcement reminiscent of pheromones in ant colonies optimization [4].

Exchange: for each of its social connection, an agent has the opportunity to exchange a piece of information. A cell is picked up randomly from the agent's memory and the other agent is informed about it. The exchange is bi-directional and the outcome of the exchange is described by the learning mechanism below.

Exploration: agents have the possibility to explore cells in the neighborhood of those that they visit. This stage is mainly intended to recover potential information loss in the other stages, thus relieving the implementation from checking that any cell was lost from the global knowledge of all the agents. Obviously $O(A)$ operations are required.

\section{Learning mechanism}

The memory of an agent is represented on Figure 1: a first buffer contains the information about locations that are either close to home or close to work (e.g. a small circular area). The second buffer called "elite" buffer (of size $E$ ) corresponds to locations that have high score values and the third buffer contains "vague" information about cells that have poor score values. When an agent informs another agent during the exchange stage, a cell is picked randomly from the total memory of the informer. At that point, the informed agent evaluates how this new cell information can potentially improve his/her plan score. This implies to evaluate the replacement of any intermediary stop by the new cell: $O\left(N^{(S-1)}\right)$ operations are needed. If the score is better than the worst solution of the elite buffer, the new cell is promoted to that buffer and it is sorted: $O(E \ln E)$ operations are needed. If the cell does not improve one of the elite plans, the cell information replaces a previous cell from the vague buffer. This has two consequences: Firstly, agents keep information that is not relevant to themselves but that might be to others in the future, hence they adopt a cooperative behavior that is not supervised. Secondly, the information in the vague buffer can be erased and lost forever. The information about cells with very low utility is more likely to be lost from the collective memory. The exploration phase allows still to recover them. So far, the computation load of a single round is $O\left(A N^{(S-1)}\right)+O(A E \ln E)+O(C)$ which is feasible for reasonable assumptions $(S \leq 3, N<50, E<50)$. However, the number of iterations is still to be determined. Note that the learning speed of the overall process depends on the greediness of the exchange which is a function of the ratio between the sizes of the elite and the vague buffers. 


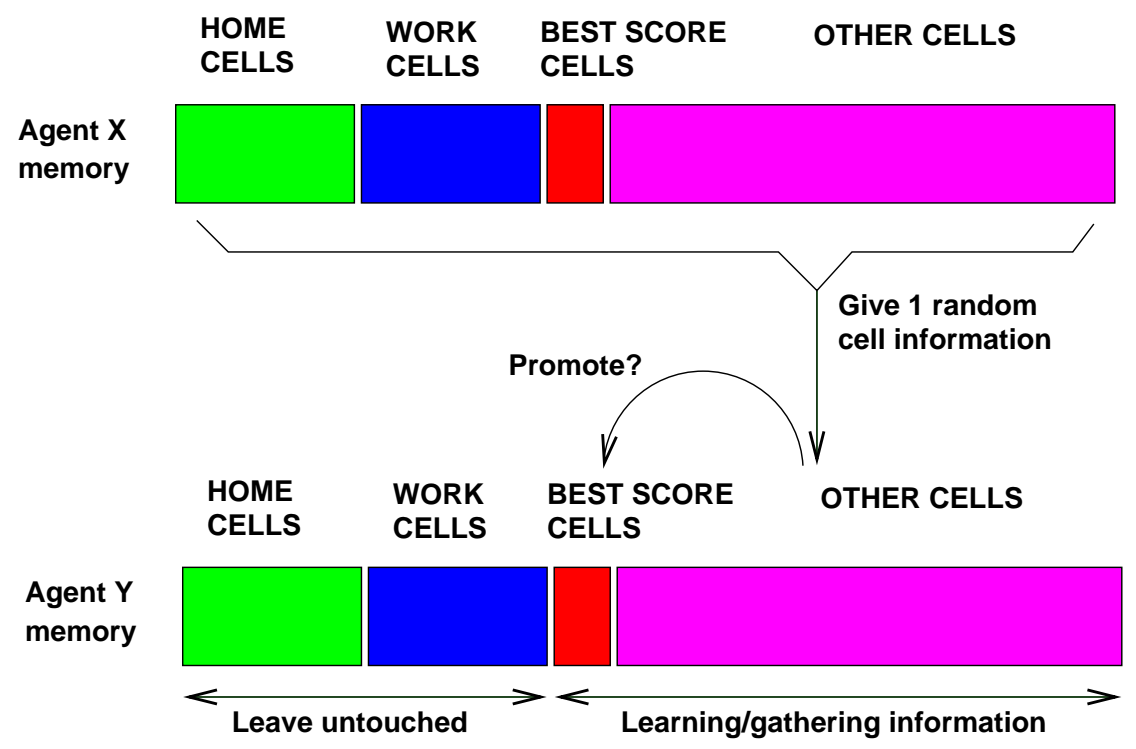

FIGURE 1. Agent memory organization and learning

\section{Social network dynamics}

Social links disappear following an exponential decay law at the end of each iteration. Initially, the social network is a random graph of degree $K$ and each social connection has the same decay time. When two agents perform activities at the same location, two situations can occur: 1 ) if there is a social connection between them, the strength of the connection is reinforced and its decay time increases 2) if not, a new social connection is created. This task requires $O(A K)$ operations for the enumeration of the social links and $O(C)$ operations to browse the cells for detecting interactions between agents. However, the detection of the existence of a social link between two given agents would require $O(A K)$ operations for each cell to browse the connections. An alternative solution would be to store the connections of a given agent in a hash table. Both solutions are costly in memory or computation time. For these reasons, we adopt a slightly different implementation which is equivalent statistically. Every connection has the same decay time but multiple redundant connections can exist between two given agents. When an agent visits a cell from his own elite buffer, the cell keeps a pointer to the agent until a second one visits the cell. A new social connection is then created between the two visitors. A hash table of visited cells and visitors needs to be maintained, which is $O(\log (C))$ costly. After an iteration, we end up with a larger number of connections that at the beginning. All of them are of equal strength but with potential redundancy (a given pair of agents can appear several times). Identifying the redundancy would be costly. Instead, we keep the total connection strength 
constant in the system by deleting connections randomly so that the total number of connections is constant.

\section{Implementation issues}

The code of the simulation has been written in Java. Input and output files use the XML file format which is well suited to variable length content such as the description of the individual plans with multiple stops. The goal being to simulate $10^{6}$ agents on a single $\mathrm{CPU}$, some performance concerns have to be taken into account.

Cells: each grid cell is stored as an individual object. With $10^{5}$ cells, it is not crucial to store the cell attributes as plain arrays. This allows to keep cell characteristics private and to have cell references. Since cells have to be often compared during the learning process to determine if a new cell is already known to an agent, it is far more efficient to use the equal operator $==$ than the default Java equals() method. This is valid as long as the cells are not dynamically allocated or cloned once the simulation starts. Cells have to keep references to agents that visit them (see the socialization stage). The average number of visits per cell is small $(O(S A / C))$ so that we can allow for the overhead of a dynamic container (e.g. vector).

Agents: it is tempting to have a dynamic container steadily increasing in size for the agent memory. However, that would completely ruin the performances and is not compatible with our assumption that only a limited number of simultaneous options can be memorized. Each cell is referenced on average by $O(A N / C)$ so that the initial coverage is sufficient to ensure that there is not any information missing about the environment.

Random numbers: a typical bottleneck of this kind of simulation is the computation of random numbers. A priori, $O(A K)$ random numbers have to be computed for each single iteration of the information exchange stage. This quickly becomes prohibitive and can be avoided by using two integer random seeds at the beginning of the exchange stage. One is used to pick a cell from the informer agent, the other to replace a cell in the memory of the informed agent. These two pointers can be simply incremented from one social link to the other since there is no correlation between social links and they are accessed in a a priori random order.

\section{Results}

The simulation is tested on a real-world example for the Zurich region for which we have available a high resolution transportation network and a land-use raster (see Figure 5). The area covers approximately a 50x50 kilometer square area where about one million inhabitants are living. The land-use utility values $R_{i}$ are generated based on census data. Random plans with 1 or 2 intermediary stops are 
generated for $10^{6}$ agents that are distributed on the area according to job and housing densities. The home to work pairs are computed using an external model written by one of the author (see [7] for the computation of the rent values presented in Figure 5). The initial social network that connects them is a random graph. Obviously, this is not realistic but we intend to evaluate only the computational feasibility in this preliminary work.

Figure 2 presents the evolution of the sum of the scores of all the agents during the iterative process. It can be seen that the process converges in a few dozens of iterations but that the choices are not optimal since the utility does not reach the maximum value obtained with a full enumeration of the alternatives. This is due to the fact that some information is lost in the process and it is recovered very slowly. Still, the value of the plateau is high enough to ensure plausible strategies for the plans. Note that the size of the memory of the agent only slightly affect the convergence properties.

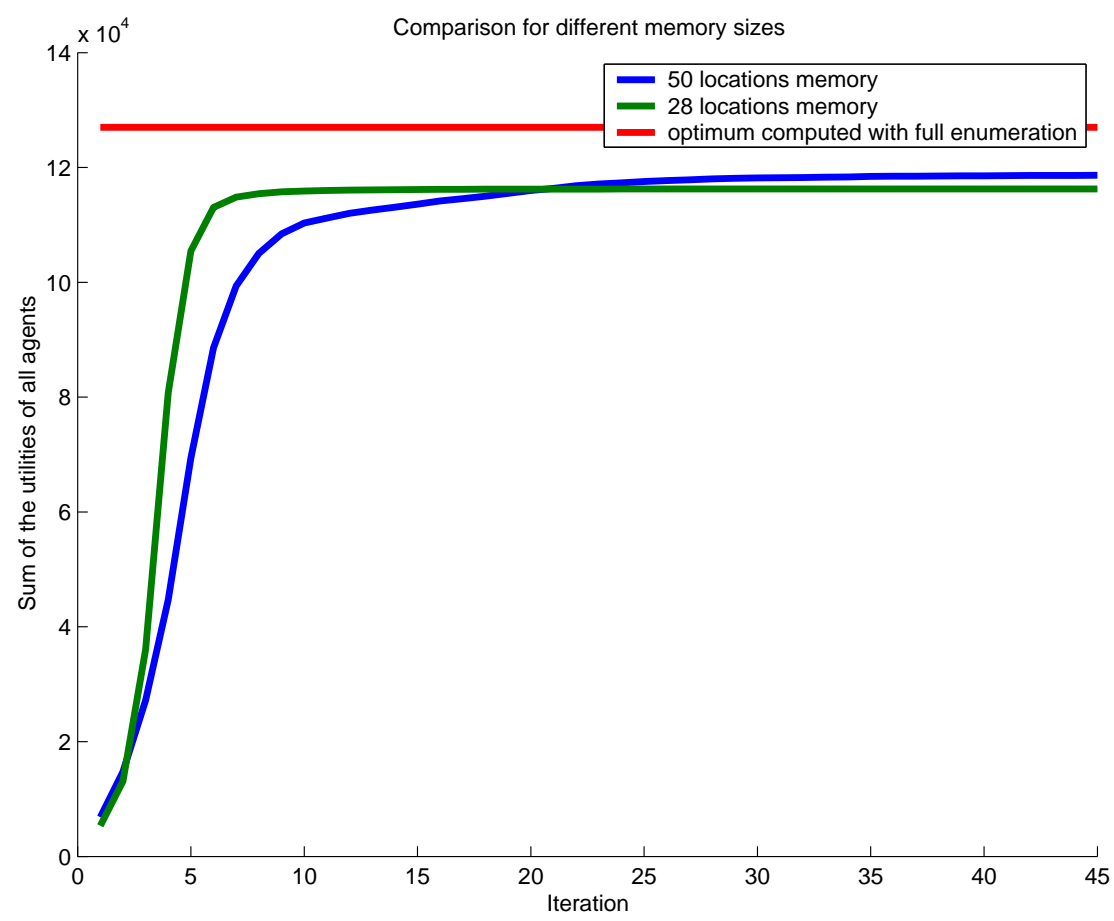

FiguRE 2. Performance of the multi-agent simulation

Figure 3 illustrates the spatial adaptation process for a single agent with a two-stop plan: home-work-leisure-shopping-home. On the first iteration, leisure $(L 1)$ and shopping $(S 1)$ are performed at the home place because the agent ignores good locations to perform these activities. In the second iteration, he/she learns 
that the location $L 2-S 2$ is a good location for one of the two activities, hence making the extra trip distance worth it. On the third iteration, the agent discover that the area around $S 3$ has a high utility for shopping. During the fourth and fifth iterations, the agent keeps shopping close to that area and only optimizes the leisure location $(L 3 \rightarrow L 4 \rightarrow L 5)$.

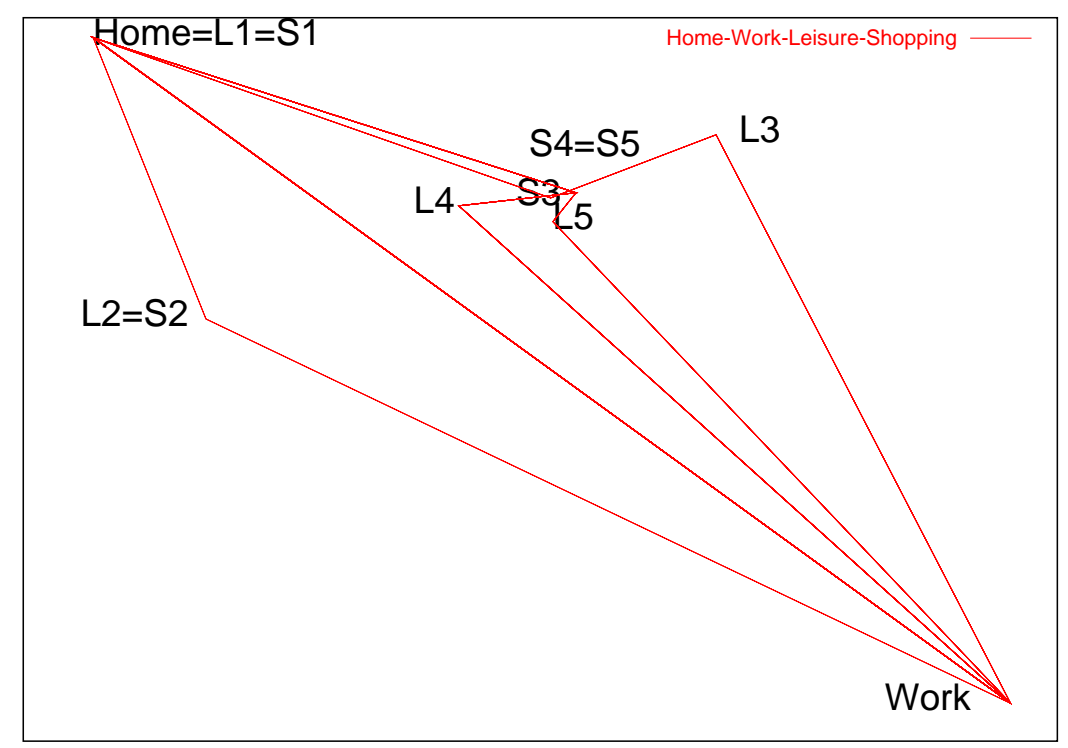

Figure 3. Adaptation of the location for a two-stop plan

Figure 4 shows the evolution of the distribution of social connections in the system. The initial condition is a random graph of degree $K=20$. On the first iteration, the distribution is roughly a $N(20,1)$ distribution. Progressively, the distributions shifts toward the left. In the end, we have a self-sustained distribution that can be approximated by a $N(13,4)$ distribution. Note that redundant connections between a given pair of agents are not identified and are therefore counted multiple times. The decrease (from 20 to 13) in the average number of social connections indicates that the spatial interaction is not sufficient, in this case, to sustain 20 connections on average. This is dependent on the properties of the land-use data such as the concentration of high-utility areas. It also depends on the total number of agents in the system. (The area under the curve is equal to the total number of connections and thus constant.)

All the experiments were done on a computer equipped with a Intel Pentium 4 clocked at $2.5 \mathrm{Ghz}$. The typical simulation performance for 100 iterations of a system with $10^{6}$ agents is below one hour of CPU time. This is for plans that have only one or two intermediary stops. In term of memory requirement, about 


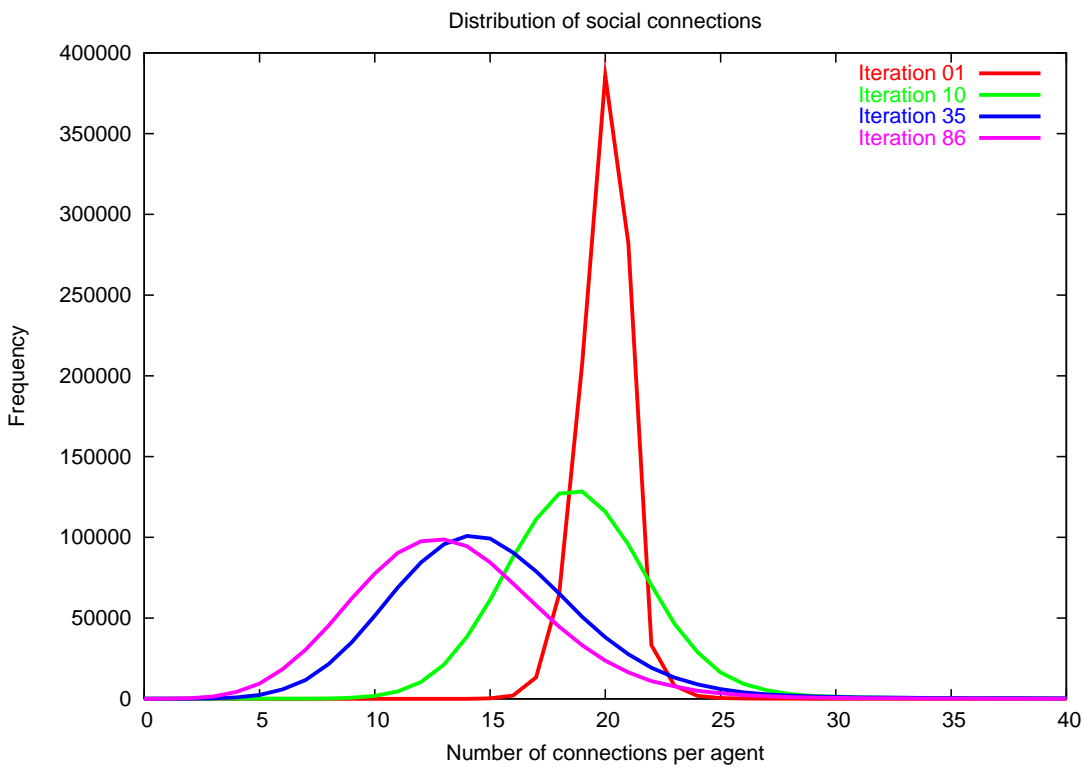

FIGURE 4. Distribution of social connections

400 Mbytes of RAM are needed. Obviously, the simulation of larger systems and more sophisticated plans with more than two stops will require to distribute the workload on several computers.

\section{Conclusions and perspectives}

Many aspects still need to be addressed to improve the realism of the model. In particular, we plan to validate the model using the results of a recent survey [9] conducted in Germany and Switzerland. The goal was to study the factors that influence leisure trips. Respondents were asked to give the location and the frequency of the visits to their five closest friends or relatives. Empirical results suggest that the purpose of an important share of leisure trips is to visit social connections. These data should, in principle, allow to compare the spatial distribution of social connections with that of our model. This preliminary work has shown that a multi-agent based approach to the location of secondary activities is technically feasible and behaviorally plausible for high resolution data sets. The fact that agents cooperate in even some simplistic way yields an important gain in term of computation workload. This has to be compared with the standard practice in Transportation Science where it is typically assumed that users are in the situation of a non-cooperative Nash equilibrium. 


\section{Acknowledgments}

The authors express their thanks to Prof. K. Axhausen and PTV AG for providing them with the data for the Zurich area. Credits go also to A. Altenhoff who wrote a first implementation of the simulation. Computer resources for the simulations were provided by the Computational Laboratory (CoLab) at ETHZ.

\section{References}

[1] T. Arentze and H. Timmermans. Representing mental maps and cognitive learning in micro-simulation models of activity-travel choice $\mathrm{dy}$ namics. In Proceedings of the meeting of the International Association for Travel Behavior Research (IATBR), Lucerne, Switzerland, 2003. See http://www.ivt.baum.ethz.ch/allgemein/iatbr2003.html.

[2] K. Axhausen, A. Zimmermann, S. Schönfelder, G. Rindsfüser, and T. Haupt. Observing the rhythms of daily life: A six-week travel diary. Transportation, 29 (2):95$124,2002$.

[3] M. Ben-Akiva and S. R. Lerman. Discrete choice analysis. The MIT Press, Cambridge, MA, 1985.

[4] E. Bonabeau, M. Dorigo, and G. Theraulaz. Swarm Intelligence : From Natural to Artificial Systems. Santa Fe Institute Studies on the Sciences of Complexity. Oxford University Press, 1999.

[5] A. de Palma and F. Marchal. Real case applications of the fully dynamic METROPOLIS tool-box: an advocacy for large-scale mesoscopic transportation systems. Networks and Spatial Economics, 2(4):347-369, 2002.

[6] T. A. Domencich and D. McFadden. Urban travel demand. In D. Jorgenson and J. Waelbroeck, editors, Urban travel demand, number 93 in Contributions to Economic Analysis. North-Holland and American Elsevier, 1975.

[7] F. Marchal. A trip generation method for time-dependent large-scale simulations of transport and land-use. Networks and Spatial Economics, forthcoming.

[8] B. Raney, N. Cetin, A. Völlmy, M. Vrtic, K. Axhausen, and K. Nagel. An agentbased microsimulation model of Swiss travel: First results. Networks and Spatial Economics, 3(1):23-41, 2003. Similar version Transportation Research Board Annual Meeting 2003 paper number 03-4267.

[9] R. Schlich, A. Simma, and K. W. Axhausen. Determinanten des Freizeitverkehrs Modellierung und empirische Befunde. Arbeitsberichte Verkehrs- und Raumplanung 190, Institut für Verkehrsplanung und Transportsysteme (IVT),ETH Zürich, 2003. See www.ivt.baug.ethz.ch.

[10] P. Waddell, A. Borning, M. Noth, N. Freier, M. Becke, and G. Ulfarsson. Microsimulation of urban development and location choices: Design and implementation of UrbanSim. Networks and Spatial Economics, 3(1):43-67, 2003. 


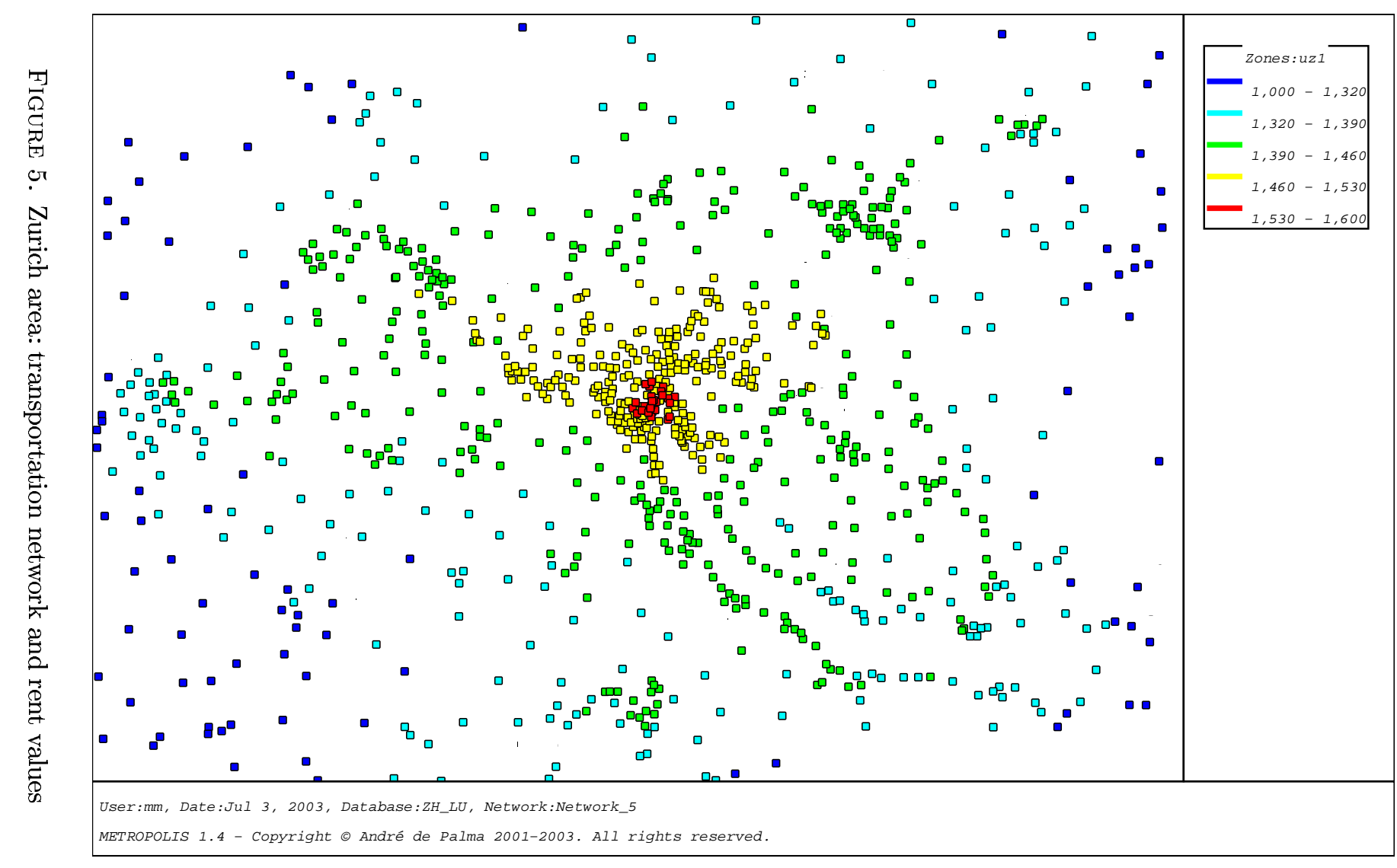


Dr. Fabrice Marchal, Computational Laboratory, Swiss Federal Institute of Technology, Hirschengraben 84, 8092 Zurich, Switzerland, Phone: +41-44-632-56-79, FAX: +41-44-632-17-03

E-mail address: marchal@inf.ethz.ch

Prof. Kai Nagel, Transport Systems Planning and Transport Telematics, Technical University Berlin, Salzufer 17-19 SG 12, 10587 Berlin, Germany, Phone: +49-30314-21382, FAX: +49-30-314-26269

E-mail address: nagel@vsp.tu-berlin.de 